\title{
Problematizando los comunes urbanos: hacia una aproximación "provincializada"
}

\author{
Problematização dos comuns urbanos: uma aproximação \\ "provincializada"
}

Problematizing Urban Commons: Towards a "Provincialised" Approach

\author{
Carlos Cámara-Menoyo [a] [D], Ramon Ribera-Fumaz [b] [D], \\ Pep Vivas i Elias [c] [D
}

[a] Oxford Brookes University, Gipsy Lane Campus, School of the Built Environment, Oxford, Reino Unido

[b] Universitat Oberta de Catalunya, Internet Interdisciplinary Institute, Laboratorio de Transformación Urbana y

Cambio Global (TURBA), Barcelona, Catalunya, España

[c] Universitat Oberta de Catalunya, Estudios de Psicología y Ciencias de la Educación, Barcelona, Catalunya, España

Cómo citar: Cámara-Menoyo, C., Ribera-Fumaz, R., \& Vivas i Elias, P. (2021). Problematizando los comunes urbanos: hacia una aproximación "provincializada". urbe. Revista Brasileira de Gestão Urbana, v.13, e20190185.

https://doi.org/10.1590/2175-3369.013.e20190185

\section{Resumen}

El discurso de los bienes comunes ha impregnado todas las facetas de la acción humana, como las ciudades, dando pie al nacimiento de comunes de todo tipo, como los urbanos. A pesar de su popularidad, los comunes urbanos siguen siendo un concepto sugerente y a la vez resbaladizo. Con el objetivo de contribuir a su comprensión y la de su potencial transformador, realizaremos un recorrido panorámico del tratamiento recibido desde la academia para confrontar las diferentes conceptualizaciones teóricas, tanto abstractas como concretas. Contrastando ambas aproximaciones, identificamos las potencialidades y límites de éstas para luego proponer una aproximación centrada en las prácticas realmente existentes; el proceso de comunalización; y el contexto en el que se producen. En particular, en un campo de estudio ampliamente dominado por enfoques y casos del norte global, el articulo aboga por "provincializar" los comunes urbanos a través de análisis situados en el sur global y, en especial, América Latina.

Palabras clave: Comunes urbanos. Teoría urbana. Ciudades.

\section{Resumo}

O discurso acerca dos bens comuns se encontra impregnado em todas as ações humanas, tal qual as cidades, originando bens comuns de todos os tipos, como os urbanos. O conceito comum urbano, apesar da popularidade, ainda segue instigante e, ao mesmo tempo, escorregadio. Com o objetivo de contribuir para a compreensão e, também, para o potencial transformador deste conceito, realizamos um percurso panorâmico do tratamento dado ao tema pela

CCM es arquitecto, doctor en Sociedad de la Información y el Conocimiento, e-mail: carlos@carloscamara.es RRF es geógrafo y ecónomo, doctor en Geografía, e-mail: rriberaf@uoc.edu

PVE es psicólogo, doctor en Psicología Social, e-mail: pvivasi@uoc.edu 
Academia, apresentando as diferentes conceituações teóricas existentes, tanto abstratas, quanto concretas. Por meio desta problematização identificamos os elementos chaves, as contradições, assim como os problemas e limitações. $O$ que nos permitiu esboçar uma nova linha de trabalho para a abordagem dos comuns urbanos, mitigando os problemas encontrados. Comparando as abordagens, o artigo identifica potencialidades e limites para, em seguida, propor uma abordagem centrada nas práticas existentes; no processo de comunalização e no contexto na qual são produzidas. Particularmente, num campo de estudo amplamente dominado por abordagens e casos do norte global, este artigo defende a "provincialização" dos comuns urbanos, por meio de análises localizadas no sul global, especialmente, na América Latina.

Palavras-chave: Comuns urbanos. Teoria urbana. Cidades.

\section{Abstract}

The commons discourse has permeated all facets of human action, like cities, and it has given rise to all kinds of commons , such as urban commons. Despite their popularity, urban commons remain a suggestive and slippery concept. To contribute to a better understanding of their features and their transformative potentialities, a panoramic on the treatment received from the academy will be made to compare the different theoretical conceptualisations, both abstract and concrete. Contrasting both approaches, this article identifies their potential and limits and then proposes an approach focused on the really existing practices; the process of communalisation; and the context in which they occur. In particular, in a field of study largely dominated by approaches and cases from the global north, the article advocates the provincialisation of urban commons through analyses located in the global south and, in particular, Latin America.

Keywords: Urban commons. Urban theory. Cities.

\section{Introducción}

El principio de siglo ha supuesto un segundo resurgimiento de los bienes comunes. Aunque este tipo de bienes o recursos datan de antiguo y en buena parte de América Latina hay una larga tradición en su uso, no ha sido hasta hace recientemente que han dejado de ser algo casi anecdótico para la academia a ser objeto de estudio de diversas disciplinas. A raíz de este resurgimiento, se han escrito numerosos artículos, libros y números especiales cubriendo diferentes aspectos de los bienes comunes (Helfrich, 2008; Hess \& Ostrom, 2007), e incluso se han fundado instituciones y publicaciones dedicadas específicamente a su estudio, como la que hoy se conoce como International Association for the Study of the Commons. Tanto es así, que autores como Frank van Laerhoven y Elinor Ostrom (2007) o Christian Laval y Pierre Dardot (2014/2015) sostienen que los estudios de los bienes comunes se han convertido en una disciplina académica por derecho propio.

Ello se debe, principalmente, a una consideración de lo común que va más allá de los tradicionales Recursos de Uso Común estudiados por Elinor Ostrom (1990) y se extiende a casi todas las facetas de la vida. Estos llamados "nuevos bienes comunes" surgen, según Charlotte Hess (2008), de la reconceptualización como bienes comunes de recursos compartidos públicamente ya existentes (calles, patios de recreo, jardines urbanos, hospitales...) y otros que se vieron favorecidos por el surgimiento de nuevas tecnologías que permitieron la captura de bienes públicos que antes no se podían capturar (Internet, datos genéticos, espacio exterior...).

Como consecuencia de lo anterior, se ha producido una reemergencia del vocabulario de los comunes en ambientes académicos pero también en círculos activistas, donde está especialmente presente en los discursos de los movimientos sociales altermundistas y por la justicia social (Seoane, 2006). Sin lugar a duda, el aumento de las estrategias comunales utilizadas por los movimientos sociales de todo el mundo en sus luchas y reivindicaciones, incluso cuando no están directamente relacionadas con recursos naturales o aspectos ambientales, ha aflorado en los últimos años. Lo común es su respuesta tanto a los efectos de la 
crisis global como a la desenfrenada agresividad de las políticas neoliberales exacerbadas por una nueva ola de políticas de austeridad y privatización surgida de ella.

Otro motivo decisivo en esta apertura de la categoría de bien común es lo atractivo que resulta el concepto. Es, aparentemente, tan autoexplicativo que resulta tentador aplicar la idea de un recurso administrado colectivamente a la mayoría de las facetas de la vida, como el conocimiento y la cultura (Hess \& Ostrom, 2007), las relaciones sociales, la música, la salud (Smith-Nonini, 2007), la genética (Scharper \& Cunningham, 2007), los recursos globales y supranacionales como la atmósfera, el espacio sideral o el ciberespacio (Stern, 2011) e incluso, tal y como desarrollaremos en este artículo, las ciudades. Estos "nuevos bienes comunes" dan una nueva dimensión a conceptos que, a pesar de no ser nuevos, nunca se habían estudiado desde la perspectiva de la acción colectiva con anterioridad (Bollier, 2007). De este modo, el subgrupo de los llamados "comunes urbanos" entroncan con discursos y reivindicaciones preexistentes como el derecho a la ciudad que proporcionan nuevas ópticas para entender, estudiar y organizar las ciudades y las relaciones sociales de sus habitantes, favoreciendo la construcción de modelos alternativos y ciudades con una mayor justicia espacial.

Sin embargo, esta "explosión" de nuevos bienes comunes de diferente índole hace más difícil que nunca definirlos. Es por ello que el presente artículo tiene por objetivo contribuir a la construcción de un marco teórico de los comunes urbanos a través de ahondar y problematizar el tratamiento que han recibido en la literatura académica reciente. En ésta, se pueden distinguir dos grandes tipos de aproximaciones. Por un lado, enfoques marcadamente teóricos -y a menudo sin trabajo empírico- que intentan conceptualizar los comunes urbanos en general. Por el otro, los estudios que exploran empíricamente -aunque teóricamente informados- comunes urbanos concretos. Contrastando ambas aproximaciones, este artículo identifica, tanto sus aspectos clave, como aquellos más contradictorios para, en última instancia, proponer una aproximación centrada en las prácticas realmente existentes; el proceso de comunalización; y el contexto en el que se producen. En particular, en un campo de estudio ampliamente dominado por enfoques y casos del norte global, proponemos "provincializar"1 los comunes urbanos con análisis situados en el sur global y, en especial, América Latina.

\section{Conceptualizando los comunes urbanos}

Resulta innegable que el concepto de común urbano es poderosamente sugerente. Parte de ello radica en que el concepto está formado por dos palabras de uso cotidiano que remiten, por un lado, a las lógicas que permitían una gestión y disfrute de bienes o recursos naturales que han existido en buena parte de la historia de muchos países, como los pastos o acequias comunales y, por otro, a la condición característica de la nueva era urbana en la que estamos inmersos actualmente. No obstante, hacer una traslación directa de esas lógicas producidas principalmente en entornos locales y rurales a entornos globales y urbanos es limitada y problemática, cuando no imposible. Tanto es así que son varios los autores que han tratado de definir qué son los comunes urbanos. Pese a todo, a día de hoy, no existe una definición compartida, pues no hay una sola forma de entenderlos: desde formas creativas de empoderamiento ciudadano a través de actuaciones de escala pequeña más o menos improvisadas, hasta formas de ejercer una interpretación del derecho a la ciudad proclamado por Henri Lefebvre para reconstruir una urbe que dé cobijo y posibilite una sociedad más justa.

Uno de los autores más citados en la literatura de los comunes urbanos es David Harvey, que vuelve a ponerlos de actualidad a partir de vincularlos con el derecho a la ciudad Lefebvriano y de hacer una lectura espacial marxista. Harvey (2012/2013) defiende que lo común no es un tipo concreto de cosas, activos, o procesos sociales, sino las relaciones sociales maleables e inestables entre un grupo social autodefinido y

\footnotetext{
${ }^{1}$ En este contexto, movilizamos el concepto en el sentido que los estudios postcoloniales lo utilizan para confrontar las visiones eurocéntricas de producción del conocimiento (ver por ejemplo Sheppard et al., 2013). En la cuarta sección del artículo profundizaremos en su definición.
} 
aquellos aspectos de su entorno social o físico considerada como crucial para su vida y subsistencia. También señala la importancia de la comunalización, entendida como la práctica social que establece una relación con los bienes comunes y que se caracteriza principalmente por ser colectiva y quedar fuera de la lógica de valores e intercambio del mercado. Para Harvey, la única opción posible consiste en que la población se auto-organice para gestionar sus propios bienes comunes, dado el contexto en el que los bienes públicos disminuyen o se convierten en un instrumento de acumulación privada y en el que el Estado se retira de su gestión.

En una línea teórica similar encontramos a Massimo de Angelis. Para él los comunes urbanos son posibilitadores de las condiciones necesarias para proporcionar justicia social, sostenibilidad y felicidad a los habitantes de las ciudades (de Angelis \& Stavrides, 2010). Estos proporcionan un nuevo discurso político, capaz de articular las múltiples luchas (normalmente descoordinadas) existentes desde hace tiempo en contra del capitalismo. Según de Angelis, los comunes urbanos son mucho más que una serie de recursos compartidos, y siempre deben de implicar los siguientes tres aspectos: un tipo de recurso común (Common Pool Resource) capaz de satisfacer una necesidad; una comunidad que comparte el recurso común y a la vez lo mantiene -y que no tiene por qué ser local ni ser homogénea-; y un tercer elemento, el más importante para él, un verbo: comunalizar (to common) que refleja el proceso social por el que el común se crea y se reproduce. En este sentido, la definición que hace de Angelis es casi idéntica a la proporcionada por Ostrom (1990) en lo que se refiere a los comunes naturales, con la salvedad del último punto de vista de entender los comunes como un proceso. Cabe señalar que, a su vez, esta idea fue introducida por primera vez por Peter Linebaugh (2008) al estudiar los comunes de la Inglaterra postfeudal y precapitalista, la cual que ha calado profundamente en el discurso de los comunes urbanos y que, como hemos visto, comparte también con Harvey.

Ida Susser y Stéphane Tonnelat (2013) comparten también esta visión positiva y transformadora de los comunes urbanos, y afirman que son una posible puerta de escape del neoliberalismo. Sin embargo, la manera de entenderlos es totalmente opuesta: ambos los conciben como movimientos sociales basados en experiencias colectivas cotidianas realizadas en espacios públicos y, a menudo, a través de artes creativas (Susser \& Tonnelat, 2013, p. 106). Para ellos, los comunes urbanos permiten visibilizar y problematizar las desigualdades y conflictos sociales de las ciudades. Siguiendo su razonamiento, los comunes urbanos cumplen con una segunda función: delinear tres de los aspectos específicos del derecho a la ciudad: el derecho a la vida diaria urbana, el derecho a la simultaneidad y a los encuentros; y el derecho a la actividad creativa. Así pues, estos autores distinguen tres tipos de comunes urbanos: los relativos a la fuerza de trabajo, al consumo colectivo y a los servicios públicos; el espacio público (que extienden más allá de calles, plazas y parques hasta llegar a lo público digital -si es que existe- de Internet), entendido como lugar de visibilización, representación y serendipia; y las expresiones artísticas en los espacios públicos, entendidas como formas de favorecer nuevos imaginarios y visiones alternativas. Esta concepción muestra claramente un hecho que se repiten a menudo entre estudiosos de comunes urbanos: la existencia de cierto solapamiento entre los bienes públicos y los comunes.

De forma similar, Stavros Stavrides plantea los comunes urbanos como los intersticios entre los espacios desde donde se ejerce la normalización espacial Foucaltiana de las clases dominantes para perpetuarse (Stavrides, 2015). Al calificarlos como umbrales, este autor destaca su condición liminar en tanto que se trata de lugares que pasan desapercibidos a ojos de la mayoría (lo cual, según él les dota de un potencial de penetración social y transformador elevados). Ello les otorga un cierto grado de poética dado que, tradicionalmente, los umbrales han sido lugares cargados de simbología ligada a la iniciación y al paso de un lugar o una situación concretos a otros distintos.

Otra línea de pensamiento la encontramos en quienes definen los comunes urbanos de forma negativa, es decir, en lugar de destacar sus características, destacan aquello a lo que se oponen: los comunes urbanos, pues, se convierten en formas de resistencia (Harvey, 2012/2013) frente a nuevas formas de cercamiento (Hodkinson, 2012; Jeffrey et al., 2012) y desposesión propiciados por las políticas neoliberales. A su vez, el 
concepto de cercamiento, alude a los cercamientos parlamentarios que terminaron con los comunes en Inglaterra, si bien los actualiza y presenta como mecanismos de desposesión a través de prácticas espaciales regidas por lógicas neoliberales que acechan a la ciudad contemporánea. Este modo de entender los cercamientos tiene sus raíces en el concepto de la acumulación primitiva de los teóricos marxistas y otorga un lugar central a los procesos de neoliberalización de las ciudades mediante un amplio abanico de procesos. Ejemplos paradigmáticos son la privatización, la mercantilización del espacio urbano y de la vida urbana o el desplazamiento a través de operaciones de gentrificación.

No obstante, otros autores han preferido centrarse en algunos aspectos concretos de los comunes urbanos para poder realizar una definición más genérica. Francesca Ferguson (2014), por ejemplo, proporciona una visión más espacial de los comunes urbanos. Para ella, se trata de espacios compartidos cuyo acceso es abierto, gestionados de forma autónoma y democrática, a modo de plataforma ciudadana que a su vez implica una forma de organización (gobernanza), un espacio y un objeto de lucha- en la que continuamente se renegocian valores sociales y políticos a través de la forma construida. Mauro CastroComa y Marc Martí-Costa (2016, p. 135) constatan, sin embargo, que la dificultad de discernir entre lo que es y lo que no es urbano reside en el hecho de que se trata de una "cuestión abierta, contextual y sobre todo política", y prefieren hablar de recursos comunes (desprendiéndose del calificativo "urbanos") para designar a aquellos recursos en los que existe una comunidad que los reclaman como tales. Es decir, ponen el énfasis en la comunidad y en los recursos compartidos. Este aspecto ya lo había mencionado David Bollier (2008) al explicitar que un común existe cuando una comunidad determinada decide gestionar un recurso de manera colectiva, poniendo énfasis en que su uso y su acceso sean equitativos y sostenibles.

Tras esta panorámica, decididamente no exhaustiva, cuya virtud es ofrecer una revisión amplia de las distintas formas de abordar el mismo fenómeno, se hacen evidentes los siguientes hallazgos. Primero, el debate sobre la conceptualización de los comunes urbanos acontece primordialmente entre académicos del norte global y en publicaciones en inglés. Si bien existen numerosas experiencias de comunes en los ámbitos hispanohablantes en general, y en Latinoamérica en particular, lo cierto es que los comunes urbanos no han sido tan estudiados, salvo por honrosas excepciones como las mencionadas de Castro-Coma y Marti Costa (2016), Helfrich (2008) o Seoane (2006) y las investigaciones de Álvaro Sevilla-Buitrago (2013).

El segundo hallazgo es que, pese al elevado grado de abstracción de las distintas visiones, es difícil encontrar puntos compartidos en los comunes urbanos más allá de los siguientes: a) Son un subgrupo de comunes cuyas singularidades específicas hacen que sea imposible trasladar directamente las mismas características de comunes paradigmáticos como los naturales; b) Poseen una dimensión que es simultáneamente material e inmaterial; c) Persiguen un beneficio común con vocación de trascender a la propia comunidad a través de incidir en la configuración de la ciudad.

El tercer y último es que, dado que la disparidad y el grado de abstracción de las distintas visiones son notables, resulta extremadamente problemático hacer una aproximación exclusivamente teórica y abstracta de un concepto tan complejo y poliédrico como los comunes urbanos. Es por ello que llevar la discusión teórica planos más concretos, prácticos y en el territorio puede aportar claves que permitan salir de este aparente cul de sac.

\section{Explorando los comunes urbanos}

En contraste con las aproximaciones anteriores, existe una extensa literatura que explora los comunes urbanos a través de casos empíricos. En ellos detectamos una serie de categorías que son consideradas como tales y que se repiten a menudo. Sin duda, la más paradigmática es la de huertos urbanos o jardines comunitarios, tal y como argumentan, entre otros, Efrat Eizenberg (2012), Ferguson (2014), Johan Colding y Sthepan Barthel (2013) o Marta Camps-Calvet et al. (2015) y lo demuestran los numerosos ejemplos que han proliferado en ciudades de todo el mundo. Tanto es así que en muchos casos parece que todos los 
huertos urbanos sean comunes urbanos y, a su vez, todo común urbano deba de tener un huerto, lo cual es una reducción tan errónea como peligrosa.

Otra práctica recurrente asociada a los comunes urbanos es la formada por las cooperativas de necesidades básicas como las de vivienda, comida, educación o sanidad (Eizenberg, 2012). Tanto es así que autores como Ana Dzokić y Marc Neelen (2015) llegan a afirmar que las numerosas cooperativas que surgieron tras la industrialización podrían considerarse como los primeros comunes urbanos, dado que suponían el principio de la construcción del Estado del bienestar a través de prácticas comunitarias que cubrían las necesidades básicas de los trabajadores. Henrik Larsen y Anders Lund Hansen (2015), por su parte, son conscientes de que entender la vivienda como un común urbano es algo cuestionable, ya que podría parecer más razonable considerarla como un bien público o simplemente un producto o mercancía, tal y como se hace a menudo. Sin embargo, alegan que la vivienda económica es también una fuerza de resistencia contra los nuevos cercamientos.

Asimismo, existe un grupo de autores que asimilan parques, plazas, calles y, en general, el espacio público a los comunes urbanos (Bruun, 2015; Ferguson, 2014; Stavrides, 2015). Por ejemplo, para Orvar Löfgren (2015) una estación de tranvía o una playa urbana son comunes urbanos; mientras que Amita Baviskar y Vinay Gidwani (2011) consideran que las escuelas públicas, los parques, las calles y los espacios públicos así como el transporte colectivo y la sanidad pública también lo son, alegando que conforman la cultura pública propia de cada ciudad. Otros prefieren matizar sus posiciones, argumentando que únicamente partes de los espacios públicos son comunes urbanos como, por ejemplo, determinados parques para perros gestionados colectivamente (Matisoff \& Noonan, 2012) o el Speaker's corner de Hyde Park (Cooper, 2006). O que deben darse determinadas condiciones para que un espacio público pueda considerarse un común urbano. En esta línea están Harvey (2012/2013) o Stavrides (2015): para ellos los espacios públicos son candidatos a ser comunes urbanos siempre que la ciudadanía se apropie de ellos para llevar a cabo una acción política que los transforme, lo cual a menudo es sinónimo de conflicto y luchas. Así, las plazas Syntagma en Atenas, Tahrir en El Cairo o Catalunya en Barcelona se convirtieron en comunes urbanos en el momento en el que la gente se concentró en ellas para expresar sus demandas políticas.

También existe literatura de casos de estudio más variados, como el caso de las guías gay de Londres y Berlín en los años 1930. Para Leif Jerram (2015), se trata de comunes ya que muestran una forma ideada por el colectivo gay para la apropiación del espacio de una ciudad que les daba la espalda, para convertirlo en lugares de ocio, de relaciones sociales y de sexo, pero también en un espacio de discusión política en el que reivindicar la posición del colectivo. Otro ejemplo es el de los residuos. Patrick Zapata y María José Zapata Campos (2015) argumentan que vertederos, como el de La Chureca en Managua, son comunes urbanos dado que albergan a una comunidad que vive en ellos y que gestiona colaborativamente los residuos como recursos que les permiten subsistir al margen del mercado.

Otros grupos de ejemplos más controvertidos serían los formado por centros sociales (incluso -o especialmente- los okupados) (Hodkinson \& Chatterton, 2006) o las comunidades exclusivas de viviendas y servicios para élites sociales (normalmente cerradas por vallas o muros -de ahí su nombre en inglés: gated communities) (LeGoix \& Webster, 2006) que tanto proliferan en el continente Americano y Asia.

Por último, están los que entienden la ciudad al completo (tanto desde el punto de vista físico -recursos, edificios e infraestructuras- como social -habitantes y relaciones sociales-) como un gran común. Hay dos justificaciones principales para tal afirmación: la primera consiste en considerar la ciudad como lugar de esperanza donde se producen las transformaciones necesarias para combatir las nuevas formas de cercamiento (Foster \& Iaione, 2015; Harvey, 2012/2013; Ramos, 2016). La segunda se basa en considerar que la multitud es hoy al proletariado lo mismo que la ciudad a la fábrica, tal y como defienden Michael Hardt y Antonio Negri (2009/2011). En otras palabras, la ciudad es la nueva fábrica en tanto que es donde se produce la desposesión y las luchas (especialmente de clase), que ya no solo afectan al proletariado, sino a la multitud. Las ciudades, por tanto, son las mayores productoras de comunes hoy en día, como lo fueron los bosques y zonas rurales anteriormente. 
Sin ánimo de clasificar exhaustivamente las prácticas de comunes urbanos presentadas en este apartado, podemos observar que dichas concepciones señalan tres dimensiones diferentes y a la vez complementarias de los comunes urbanos:

a) El común urbano como grupo o comunidad que comparten espacios de la ciudad y que se autoorganizan y plantean formas alternativas de experimentar la urbe y basadas en una concepción del espacio público y colectivo como un común;

b) El común urbano como espacio de posibilidad, como lugar de planteamiento a lo "alternativo" en relación con la normalidad y la cotidianidad urbana;

c) El común urbano como acción colectiva que se construye mediante la acción de la ciudadanía. El común urbano no queda instituido y no tiene agencia como tal hasta que no queda construido y es practicado colectivamente.

Finalmente, cabe destacar, que ante la gran diversidad de ejemplos entre el norte y el sur global, la presencia de investigaciones sobre comunes urbanos en América Latina o en español o portugués es muy limitada.

\section{Problematizando los comunes urbanos}

Contrastando las dos aproximaciones anteriores (abstracta y empírica), observamos como casi la totalidad de académicos atribuyen a los comunes urbanos cualidades positivas basadas en los principios de cooperación, acceso abierto y búsqueda de beneficio social (que no económico) que se oponen al modelo capitalista imperante a través de la construcción de realidades y espacios urbanos alternativos y transformadores. Haciendo un esfuerzo por sintetizar los aspectos compartidos señalados en las secciones anteriores, nos aventuramos a realizar la siguiente propuesta de definición de consenso:

Los comunes urbanos son prácticas sociales localizadas y organizadas alrededor de lugares o recursos singulares. A su vez, dichos lugares o recursos son gestionados colectivamente por una comunidad autoorganizada, cuya motivación es dar respuesta a un conjunto de reivindicaciones urbanas e históricas que tienen sus raíces en la ciudad como oposición de cercamientos de todo tipo. Al hacerlo, construyen nuevas formas de ejercer la democracia desde la práctica (esto es, no limitadas a votar cada 4 años), regidas por lógicas de funcionamiento ajenas al capitalismo y mediante la transformación urbana y la acción colectiva de una comunidad de la que dependen y, a su vez, fortalecen y cohesionan. En última instancia, dada su condición de límite, plantean una alternativa a la dualidad entre lo privado y lo público que pone en crisis el modelo productivo imperante de las ciudades.

Sin duda, se trata de una propuesta sugerente que abre un gran número de oportunidades y campo de estudio basado en los mecanismos de oposición y alternativas que promueven los comunes urbanos y su potencial transformador para con las ciudades y las sociedades. No obstante, si bien esta definición funciona muy bien para describir muchos de los casos ejemplificados en el apartado anterior, muestra lagunas en determinados casos extremos. Un enfoque como ese es problemático porque, de nuevo, lleva inevitablemente a plantear preguntas dicotómicas que no pueden responderse de forma categórica. Ejemplo de dichas preguntas serían si determinadas tipologías (bibliotecas, centros cívicos, hackerspaces, centros okupados, o cooperativas de cualquier tipo, por poner algunos ejemplos) son siempre comunes urbanos; si es necesaria una dimensión física para ser un común urbano o, por el contrario, algo eminentemente digital puede serlo también; o si los comunes urbanos deben surgir siempre desde la base y, por tanto, quedan excluidas iniciativas promovidas por la Administración o por el sector privado en forma de emprendizaje social, entre otras preguntas por el estilo. No solo eso, una visión artificialmente homogeneizadora como esta camufla el hecho de que existen grandes diferencias en lo que se considera, dentro de la academia, un común urbano.

Incluso a pesar de tratarse de un campo aparentemente acotado, ha quedado patente que no existe un cuerpo teórico consolidado sobre comunes urbanos. Ello hace que sea habitual tomar una posición selectiva 
en exceso (y, consecuentemente, parcial) o demasiado genérica. Así pues, hemos visto el esfuerzo que han hecho distintos académicos por intentar definir los comunes urbanos o identificar sus aspectos definitorios. Sin embargo, estas definiciones suelen hacerse ad-hoc para describir determinados casos concretos atendiendo únicamente a algunos de esos requisitos u ofreciendo una reinterpretación laxa de conceptos clave, como la dicotomía de público y común.

En este sentido, es relativamente frecuente encontrar referencias a espacios y bienes públicos como sinónimos de bienes comunes. Tal y como hemos visto, hay autores que consideran que determinados espacios o, directamente, el espacio público son comunes urbanos en tanto a que su acceso es abierto. Sin duda, se trata de una concepción muy limitada y problemática. Primero, porque el espacio público no es necesariamente un espacio abierto a todos los públicos. A modo de ejemplo las residencias de los jefes de estado suelen ser públicas pero su acceso está restringido a unas pocas personas. Segundo, aunque aceptáramos el acceso abierto universal, incluso espacios tan controvertidos como centros comerciales podrían llegar a considerarse comunes urbanos cuando en realidad son una expresión física del capitalismo totalmente opuesta a la lógica del común.

Esta concepción de bienes o espacios comunes es especialmente frecuente en autores de países donde el Estado del bienestar y la cultura de lo público no están tan enraizadas frente a la cultura de lo privado, donde espacios como centros comerciales hacen las veces de espacios públicos en tanto que son lugares de pública concurrencia, están vinculados a actividades de ocio e incluso cultura y permiten la socialización entre grupos de personas. Ante esta situación es habitual contraponer estos espacios a otros que no están ligados a entidades privadas o al consumo (dos aspectos totalmente contrarios a la ideología de los comunes) como parques, calles o plazas. Por ello, algunos autores han tratado de distinguir entre común, privado y público como Stavrides (2015, p. 10-11), en función de quiénes crean y gestionan los espacios: ciudadanía (comunes), autoridad (públicos), o individuos o entidades económicas (privados).

Sin embargo, esto también puede resultar problemático. Por ejemplo, sistemas urbanísticos como el español o italiano contemplan y tipifican los bienes de titularidad comunal, diferenciándolos de los de titularidad pública o privada. Pese a que el debate entre bien público y bien común es pertinente, es también tramposo en cuanto a que los comunes urbanos nada tienen que ver con la titularidad del suelo, puesto que tradicionalmente se han opuesto a ella ${ }^{2}$. Sea como fuere, es importante dejar constancia de que ni todos los bienes públicos son bienes comunes ni todos los bienes comunes se producen en espacios públicos.

Ocurre algo similar con determinadas tipologías que son aceptadas como comunes urbanos por defecto. Tal es el caso de los huertos urbanos o, especialmente, las cooperativas. Estas últimas son a menudo equiparadas a comunes dado que su sistema de toma de decisiones y gestión es colectiva o porque muchos de los comunes urbanos constituidos legalmente optan por esa figura jurídica. Sin embargo, en la mayoría de países, las cooperativas son un régimen legal y aunque pueden tener fines sociales que vayan más allá del lucro o incluso partir de reivindicaciones, no tiene por qué ser siempre así. Por tanto, de nuevo, ni todas las cooperativas son comunes, ni todos los comunes tienen que constituirse en cooperativas.

Tampoco basta con que exista una comunidad: bajo este paraguas podrían entenderse cualquier tipo de asociación, desde una junta de accionistas, a un club de caza, pasando por una familia, unas comunidades que difícilmente pueden considerarse comunes urbanos en sí mismos. Incluso el hecho de oponerse a cercamientos de todo tipo es insuficiente: existen numerosos movimientos altermundistas e incluso movimientos sociales con esa misma motivación y, sin embargo, eso no les convierte automáticamente en comunes urbanos. Por consiguiente, tanto o más importante que identificar los aspectos definitorios de los comunes urbanos es comprender que no es suficiente con que se cumpla uno de ellos, sino que deben cumplirse todos y cada uno de ellos a la vez. El hecho de entender estos elementos por separado no es únicamente falaz sino que lleva a la siguiente consideración: la perversión y consiguiente pérdida de relevancia del concepto.

\footnotetext{
${ }^{2}$ Para mayor información al respecto remitimos a León Casero \& Cámara-Menoyo (2020), en el que exploramos las diferencias jurídicas entre los distintos regímenes de propiedad y su relación con los comunes urbanos.
} 
Como advierte Harvey (2012/2013), existe un riesgo real de que los comunes urbanos sean capitalizados mediante la apropiación de su discurso de modo que se pervierta el concepto de tal modo que acabe siendo algo muy similar a aquello a lo que se oponen. El ejemplo más claro de perversión del concepto y apropiación por parte del lenguaje capitalista es el de calificar a las comunidades de acceso restringido o condominios cerrados como comunes urbanos, algo que el mismo Harvey (2012/2013, p. 113) califica de "desfachatez" y que Elisabeth Blackmar (2006) considera la verdadera "tragedia" de los comunes. Si bien es cierto que proporcionan servicios a una comunidad, entendemos que no se trata de comunes urbanos porque, más allá de servir a una comunidad formada por una élite económica, no cumplen ningún otro de los hechos diferenciales de los comunes urbanos que hemos identificado anteriormente.

Otros ejemplos de mal llamados comunes urbanos son los proyectos de emprendedores sociales y empresas de la economía colaborativa (sharing economy) como AirBnB o Uber, que ven en los derechos sociales o en el consumo colaborativo un nicho de mercado. Así pues, aunque hay quienes defienden que dichas empresas están basadas en los principios de los comunes (Botsman \& Rogers, 2010) o que el hecho de que el consumo colaborativo sea entre pares (peer to peer) es condición suficiente para calificarlo como comunes y atribuirles sus características positivas (Dillahunt \& Malone, 2015), no son menos quienes argumentan que se trata de algo completamente distinto. Difícilmente pueden tildarse de iniciativas ciudadanas a las propuestas realizadas por grandes multinacionales o startups que buscan su nicho de mercado a la vez que contribuyen a acrecentar la brecha entre clases (Morozov, 2014) y que da lugar a un nuevo modelo de capitalismo "de plataforma" (Srnicek \& De Sutter, 2017) basado en poner en contacto a personas que ofrecen un servicio o mercancía para comprar o alquilar con personas que los necesitan, a cambio de cobrar una comisión por cada transacción realizada entre ellas, lo cual se traduce en una forma de autoexplotación por parte de iguales.

Sin embargo, sería ingenuo pensar que las únicas formas de tergiversación del lenguaje de los comunes vienen exclusivamente de fuera de su entorno: la falta de un cuerpo teórico sólido contribuye a concepciones ad-hoc por parte de estudiosos o activistas de los comunes urbanos que en algunos casos son tan particulares y parciales que contribuyen a trivializar el concepto. En el mejor de los casos, existe el riesgo de que se convierta en un mero rebranding de iniciativas que hace tiempo que se vienen dando desde hace años, como los movimientos sociales y cooperativistas. En el peor, de convertirlo en una anécdota irrelevante.

\section{Provincializando los comunes urbanos}

Debido a las problemáticas expuestas, consideramos necesaria una nueva aproximación al concepto de los comunes urbanos que permita salir del punto muerto en el que nos encontramos. Dicha aproximación debería de abordar directamente los siguientes aspectos principales: las prácticas realmente existentes; el proceso de comunalización; y el contexto en el que se producen.

Ha quedado patente que una aproximación exclusivamente teórica no es capaz de explicar todas las particularidades de una realidad extremadamente variable, compleja y dúctil. A su vez, existe una gran cantidad de iniciativas por todo el planeta calificadas como comunes urbanos. Dichas prácticas son muy diversas y, habitualmente, se producen al margen de círculos académicos. Es por ello que abogamos por incorporar dichos saberes prácticos provenientes del terreno mediante la utilización de metodologías basadas en el trabajo de campo relativamente prolongado en el tiempo, como pueden ser las etnografías o los casos de estudio, atendiendo especialmente a las tres dimensiones identificadas anteriormente tras la revisión de literatura: comunidad, espacio y acción colectiva. Esto no es óbice para ignorar la literatura existente, sino que consideramos debería complementarla para suplir las carencias señaladas anteriormente.

En segundo lugar, pese a la existencia de autores que señalan lo procesual como aspecto definitorio de los comunes urbanos, lo cierto es que no se suele abundar en ello ni suele ser objeto de estudio. Hasta ahora, la tendencia habitual consiste en trabajar con uno o pocos casos de estudio de forma aislada, 
considerándolos fenómenos endógenos y estáticos. Si bien esta aproximación permite llegar a un elevado grado de profundidad para los casos estudiados, presenta limitaciones a la hora de construir una teoría que sea capaz de explicar un fenómeno tan diverso y complejo a partir de unos pocos casos aislados. No solo eso, lejos de describir un proceso, son una instantánea de un momento que favorecen narrativas que sugieren que existe un principio y un fin en los comunes urbanos, algo por demostrar. Es por ello que consideramos necesario considerar la variable temporal para estudiar cómo evolucionan estos en el tiempo, y considerar los casos de estudio como nodos integradores de una red interrelacionada y viva que comprenda, además, nodos externos y tan variados como son otros comunes urbanos o agentes como la Administración pública, entre otros.

Finalmente, consideramos especialmente relevante, a la vez que habitualmente descuidado, la incorporación del contexto en el que se producen los comunes urbanos como elemento indispensable para su comprensión. Lejos de ser un ethos homogéneo que se implanta de la misma manera en cualquier lugar del mundo, los comunes urbanos variarán necesariamente en función de su contexto geopolítico y temporal. No se trata tanto (o únicamente) de que la noción de común urbano cambie de un lugar a otro. Se trata más bien de que, dado que cada común urbano es una lucha o reivindicación que responde a una problemática concreta, esta será necesariamente distinta en función de dónde esté situada. Así pues, el hecho de no tener cubiertas las necesidades más básicas puede hacer que, en Managua, un vertedero como el de la Chureca sea considerado un común urbano por algunos autores, algo que seguramente sería impensable en Dinamarca. Por otro lado, en países donde el Estado del bienestar no es tan fuerte y, sin embargo, existe una economía fuerte que permite la existencia de una clase media o alta relativamente numerosa, pueden existir comunes urbanos que en otros contextos serían asimilables a servicios públicos o simplemente se producirían en alguno de los abundantes parques o plazas públicas de países donde la cultura de lo público está más enraizada.

Pero no solo la problemática a resolver es distinta en función del contexto, sino que la forma en que se materializarán los comunes urbanos como herramienta al servicio de las comunidades locales para hacerle frente también variará considerablemente en función del mismo. Incluso cuando las problemáticas puedan ser similares, los resultados podrían ser muy dispares dado que también son diferentes el tipo de comunidades que gestionan los comunes, los recursos de los que disponen, las leyes a las que están sujetos, el tipo de relación que pudieran tener con las distintas Administraciones (especialmente la local) o el trasfondo cultural en el que ocurren. Tal y como Kate D. Derickson (2015, p. 651) apunta, "el acto de teorizar lo urbano y, por asociación, teorizar las posibilidades políticas, está fundamentalmente moldeado y limitado por las tradiciones intelectuales y políticas sobre las que se basan y en los ejemplos empíricos en los que se sustentan." Por ello es necesario abrir la exploración de los comunes urbanos a diferentes tradiciones y ejemplos fuera del norte global y ponerlos en diálogo con la literatura existente.

En resumen, proponemos "provincializar" el análisis de los comunes urbanos en frente a recetas universalistas. Para Helga Leitner y Eric Sheppard (2016, p. 230), provincializar significa "trastocar las normas sobre lo que pensamos: retar las explicaciones monistas para subrayar cómo están moldeadas a sus orígenes 'locales' y hacerse preguntas de cómo viajan”. Es por ello, que Jennifer Robinson y Ananya Roy (2016, p. 182) hablan de "urbanismos", para "significar 'teoría' (siempre una multiplicidad) como la proliferación de proyectos imaginativos inspirados por y productivos de gran diversidad de experiencias urbanas". Así pues, incorporando miradas de diferentes partes del sur global, desde diversos enfoques y posiciones que podemos entender y confrontar los comunes urbanos. No se trata de crear teorías universales, sinó de considerar las conexiones entre lugares, comunes y comunalización viajando a través de la diferencia (Peake, 2016). Por tanto, provincializar los comunes urbanos no solo ofrecería una mirada más diversa que se traduciría en una mejor explicación del fenómeno, sino que además permitiría salvar una de las carencias detectadas en el análisis de la literatura: la dominancia excesiva de la literatura anglosajona en la construcción del marco teórico y su correspondiente sesgo. 


\section{Conclusiones}

El presente artículo reconoce el renovado auge de los comunes en general y los comunes urbanos en particular, tanto en ámbitos activistas como académicos. Es desde este último grupo desde donde se está teorizando para construir un nuevo marco teórico de lo común que pueda aplicarse a nuevos y variados ámbitos y contextos. Tras una panorámica de la literatura sobre el común, hemos podido plantear una definición de consenso sobre los comunes urbanos a partir de la identificación de una serie de características compartidas. Dicha definición tiene la virtud de ser lo suficientemente genérica como para incorporar las distintas visiones estudiadas, a la vez que es lo suficientemente concreta para incluir prácticas variadas que comparten la lógica del común. Sin embargo, y a pesar de sus virtudes, reconocemos también las limitaciones de dicha definición. Ello se debe, principalmente a que el marco teórico de los comunes urbanos está todavía en ciernes y adolece de una extrema diversidad de enfoques que, frecuentemente, llegan a ser dispares. Estas divergencias son especialmente patentes en el momento de saltar de unos discursos extremadamente teóricos y abstractos a las prácticas comunitarias realmente existentes, mostrando una desconexión total entre ambas dimensiones.

Consecuentemente, sostenemos que el enfoque actual de los estudios sobre los comunes en general, y los comunes urbanos en particular, puede llegar a ser contraproducente, tanto para la disciplina como para el propio objeto de estudio, siendo evidente el riesgo de vaciado y apropiación de su significado. Es por ello que reivindicamos la necesidad de construir colectivamente un marco teórico riguroso, coherente, articulado y holístico que no dé la espalda a una realidad extremadamente poliédrica, compleja y diversa. En la parte final del documento hemos desarrollado una posible línea de trabajo en esa dirección. Nuestra propuesta consiste en apostar por una aproximación que no sea meramente abstracta y universalista, sino que tenga en cuenta las prácticas realmente existentes, atendiendo a lo relacional y procesual frente al estudio de casos aislados, y, especialmente, ofreciendo un enfoque provincializado, es decir, que considere el contexto como elemento definitorio y como oportunidad para explicar fenómenos formalmente distintos pero con lógicas compartidas. Si bien no era objeto del presente artículo ponerla en práctica, consideramos suficientemente justificadas las ventajas de dicha aproximación, a la vez que sienta las bases para una agenda de investigación de los comunes urbanos plural en las áreas de habla portuguesa y española en América Latina y en el resto del mundo3.

\section{Referencias}

Baviskar, A., \& Gidwani, V. (2011). Urban Commons. Economic and Political Weekly, XLVI(50), 42-43.

Blackmar, E. (2006). Appropriating the «Commons»: The tragedy of Property Rights Discourse. En S. M. Low \& N. Smith (Eds.), The Politics of Public Space (1aㅡ ed., pp. 49-80). New York, Abingdon: Routledge.

Bollier, D. (2007). The Growth of the Commons Paradigm. En E. Ostrom \& C. Hess (Eds.), Understanding Knowledge as a Commons: From Theory to Practise (1 ${ }^{\mathrm{a}}$ ed., pp. 27-40). Londres: MIT Press.

Bollier, D. (2008). Viral Spiral: How the Commoners Built a Digital Republic of Their Own (1ํㅗ ed.). New York: The New York Press.

Botsman, R., \& Rogers, R. (2010). What's mine is yours: The rise of collaborative consumption (1aㅡ. ed.). New York: Harper Business.

Bruun, M. H. (2015). Communities and the commons. En C. Borch \& M. Kornberger (Eds.), Urban Commons: Rethinking the City (1ํㅡㄹ ed., pp. 151-170). Abingdon, New York: Routledge.

${ }^{3}$ Un ejemplo de ello podemos encontrarlo en Cámara-Menoyo et al. (2021) (En prensa, publicación esperada en septiembre 2021) 
Cámara-Menoyo, C., Vivas Elias, P., Ribera-Fumaz, R., \& León Casero, J. (2021 -en prensa-). Hacia una agenda urbana del común: Un estudio de caso de las reivindicaciones políticas de los comunes urbanos en Barcelona. EURE - Revista de Estudios Urbanos Regionales, 48(143).

Camps-Calvet, M., Langemeyer, J., Calvet-Mir, L., Gómez-Baggethun, E., \& March, H. (2015). Sowing Resilience and Contestation in Times of Crises: The Case of Urban Gardening Movements in Barcelona. Partecipazione e Conflitto, 8(2), 417-442. https://doi.org/10.1285/i20356609v8i2p417.

Castro-Coma, M., \& Martí-Costa, M. (2016). Comunes urbanos: De la gestión colectiva al derecho a la ciudad. Revista EURE Revista de Estudios Urbano Regionales, 42(125), 131-153. https://doi.org/10.4067/S0250-71612016000100006.

Colding, J., \& Barthel, S. (2013). The potential of 'Urban Green Commons' in the resilience building of cities. Ecological Economics, 86, 156-166. https://doi.org/10.1016/j.ecolecon.2012.10.016.

Cooper, D. (2006). "Sometimes a community and sometimes a battlefield": From the comedic public sphere to the commons of Speakers' Corner. Environment and Planning D: Society and Space, 24(5), 753-775. https://doi.org/10.1068/d1004.

de Angelis, M., \& Stavrides, S. (2010, junio). On the Commons: A Public Interview with Massimo De Angelis and Stavros Stavrides (An Architektur) [E-flux]. Recuperado el 11 de diciembre de 2020, de http://www.e-flux.com/journal/on-thecommons-a-public-interview-with-massimo-de-angelis-and-stavros-stavrides/

Derickson, K. D. (2015). Urban geography I: Locating urban theory in the 'urban age'. Progress in Human Geography, 39(5), 647-657. https://doi.org/10.1177/0309132514560961

Dillahunt, T. R., \& Malone, A. R. (2015). The Promise of the Sharing Economy among Disadvantaged Communities. Proceedings of the 33rd Annual ACM Conference on Human Factors in Computying Systems, 2285-2294. https://doi.org/10.1145/2702123.2702189.

Dzokić, A., \& Neelen, M. (2015). Instituting Commoning. Footprint, 9(1), 21-34.

Eizenberg, E. (2012). Actually Existing Commons: Three Moments of Space of Community Gardens in New York City. Antipode, 44(3), 764-782. https://doi.org/10.1111/j.1467-8330.2011.00892.x.

Ferguson, F. (Ed.). (2014). Make_Shift City renegotiating the urban commons (1 ${ }^{\mathrm{a}}$ ed.). Berlin: Jovis.

Foster, S., \& Iaione, C. (2015). The City as a Commons. Yale Law and Policy Review, 34(2), 281-349. http://dx.doi.org/10.2139/ssrn.2653084.

Hardt, M., \& Negri, A. (2011). Commonwealth: El proyecto de una revolución del común (R. Sánchez Cedillo, Trad.). Madrid: Akal. (Obra original publicada en 2009)

Harvey, D. (2013). Ciudades Rebeldes: Del derecho de la ciudad a la revolución urbana (J. Madariaga, Trad.). Madrid: Akal. (Obra original publicada en 2012)

Helfrich, S. (Ed.). (2008). Genes, Bytes y Emisiones, Bienes Comunes y Ciudadanía. San Salvador: Ediciones Böll: Fundación Heinrich Böll.

Hess, C. (2008). Mapping the New Commons. (SSRN Scholarly Paper ID 1356835). Social Science Research Network. https://doi.org/10.2139/ssrn.1356835

Hess, C., \& Ostrom, E. (Eds.). (2007). Understanding knowledge as a commons: From theory to practice. Cambridge: MIT Press. Hodkinson, S. (2012). The new urban enclosures. City, 16(5), 500-518. https://doi.org/10.1080/13604813.2012.709403. Hodkinson, S., \& Chatterton, P. (2006). Autonomy in the city? City, 10(3), 305-315. https://doi.org/10.1080/13604810600982222. Jeffrey, A., McFarlane, C., \& Vasudevan, A. (2012). Rethinking Enclosure: Space, Subjectivity and the Commons. Antipode, 44(4), 1247-1267. https://doi.org/10.1111/j.1467-8330.2011.00954.x

Jerram, L. (2015). The false promise of the commons: Historical fantasies, sexuality and the «really-existing» urban common of modernity. En C. Borch \& M. Kornberger (Eds.), Urban commons: Rethinking the city (pp. 47-67). Abindgon, New York: Routledge.

Larsen, H. G., \& Hansen, A. L. (2015). Commodifying Danish Housing Commons. Geografiska Annaler: Series B, Human Geography, 97(3), 263-274. https://doi.org/10.1111/geob.12080. 
Laval, C., \& Dardot, P. (2015). Común: Ensayo sobre la revolución en el siglo XXI (A. Díez, Trad.). Gedisa. (Obra original publicada en 2014)

LeGoix, R., \& Webster, C. (2006). Gated communities, sustainable cities and a tragedy of the urban commons. Critical Planning, 13, 41-62.

Leitner, H., \& Sheppard, E. (2016). Provincializing Critical Urban Theory: Extending the Ecosystem of Possibilities. International Journal of Urban and Regional Research, 40(1), 228-235. https://doi.org/10.1111/1468-2427.12277

León Casero, J., \& Cámara-Menoyo, C. (2020). La precariedad de los comunes urbanos frente al derecho público europeo. ACME: An International Journal for Critical Geographies, 19(3), 726-743.

Linebaugh, P. (2008). The Magna Carta manifesto: Liberties and commons for all. Berkeley: University of California Press.

Löfgren, O. (2015). Sharing an atmosphere: Spaces in urban commons. En C. Borch \& M. Kornberger (Eds.), Urban commons: Rethinking the city (pp. 68-91). Abindgon, New York: Routledge.

Matisoff, D., \& Noonan, D. (2012). Managing contested greenspace: Neighborhood commons and the rise of dog parks. International Journal of the Commons, 6(1), 28. https://doi.org/10.18352/ijc.299.

Morozov, E. (2014, septiembre 28). Don't believe the hype, the «sharing economy» masks a failing economy. The Guardian.

Ostrom, E. (1990). Governing the commons: The evolution of institutions for collective action. Cambridge: Cambridge University Press.

Peake, L. (2016). The Twenty-First-Century Quest for Feminism and the Global Urban. International Journal of Urban and Regional Research, 40(1), 219-227. https://doi.org/10.1111/1468-2427.12276.

Ramos, J. M. (Ed.). (2016). The City as a Commons - A Policy Reader. Melbourne: The Commons Transition Coalition.

Robinson, J., \& Roy, A. (2016). Debate on Global Urbanisms and the Nature of Urban Theory. International Journal of Urban and Regional Research, 40(1), 181-186. https://doi.org/10.1111/1468-2427.12272.

Scharper, S. B., \& Cunningham, H. (2007). The genetic commons: Resisting the neo-liberal enclosure of life. En D. M. Nonini (Ed.), The global idea of «the commons» (1ª ed., pp. 43-65). New York: Berghahn Books.

Seoane, J. (2006). Movimientos sociales y recursos naturales en América Latina: Resistencias al neoliberalismo, configuración de alternativas. Sociedade e Estado, 21(1), 85-107. https://doi.org/10.1590/S0102-69922006000100006.

Sevilla-Buitrago, A. (2013). ¿Planificar los comunes? Autogestión, regulación comunal del suelo y su eclipse en la Inglaterra precapitalista. Scripta nova: revista electrónica de geografía y ciencias sociales, XVII(442). Recuperado el 11 de diciembre de 2020, de http://www.ub.es/geocrit/sn/sn-442.htm.

Sheppard, E., Leitner, H., \& Maringanti, A. (2013). Provincializing Global Urbanism: A Manifesto. Urban Geography, 34(7), 893-900. https://doi.org/10.1080/02723638.2013.807977.

Smith-Nonini, S. (2007). Conceiving the health commons: Operationalizing a «right» to health. En D. M. Nonini (Ed.), The global idea of «the commons» (1ํㅗㄹ ed., pp. 115-135). New York: Berghahn Books.

Srnicek, N., \& De Sutter, L. (2017). Platform capitalism. Cambridge: Polity.

Stavrides, S. (2015). Common Space as Threshold Space: Urban Commoning in Struggles to Re-Appropriate Public Space. Footprint, 9(1), 9-20.

Stern, P. C. (2011). Design principles for global commons: Natural resources and emerging technologies. International Journal of the Commons, 5(2), 213-232. https://doi.org/10.18352/ijc.305.

Susser, I., \& Tonnelat, S. (2013). Transformative cities: The three urban commons. Focaal, 2013(66), 105-121.

https://doi.org/10.3167/fcl.2013.660110. 
van Laerhoven, F., \& Ostrom, E. (2007). Traditions and Trends in the Study of the Commons. International Journal of the Commons, 1(1), 3-28. https://doi.org/10.18352/ijc.76.

Zapata, P., \& Zapata Campos, M. J. (2015). The myth of the urban commons. En C. Borch \& M. Kornberger (Eds.), Urban commons: Rethinking the city (1 ${ }^{\mathrm{a}}$ ed., pp. 92-108). Abingdon, New York: Routledge.

Editor: Paulo Nascimento Neto

Recibido: 18 jul. 2019

Aprobado: 03 nov. 2020 